\title{
INFLUENCIA DE LA APLICACIÓN DE BIOSÓLIDOS SOBRE EL SUELO, LA MORFOLOGÍA Y PRODUCTIVIDAD DEL CULTIVO DE CANAA DE AZUCAR
}

\section{INFLUENCE OF BIOSOLIDS APLICATION ON SOIL, PLANT AND QUALITY AND PRODUCTIVITY OF SUGARGANE CROP}

\author{
Patricia Torres-Lozada ${ }^{1}$, Jorge Antonio Silva-Leal ${ }^{2}$, Brayan Alexis Parra-Orobio ${ }^{3}$, Viviana Cerón-Castro ${ }^{4}$, Carlos Arturo Made- \\ ra-Parra ${ }^{5}$
}

\begin{abstract}
${ }^{1}$ Ingeniera Sanitaria, Magíster y Doctora en Ingeniería Civil énfasis en Hidráulica y Saneamiento. Escuela de Ingeniería de Recursos Naturales y del Ambiente-EIDENAR, Universidad del Valle, calle 13 \# 100-00, Cali, Colombia, e-mail: patricia. torres@correounivalle.edu.co; ${ }^{2}$ Ingeniero de Producción Biotecnológica, Magíster y Doctor en Ingeniería énfasis en Ingeniería Sanitaria y Ambiental. Grupo de Investigación Instrumentación Electrónica, Industrial y Ambiental-GIEIAM, Facultad de Ingeniería, Universidad Santiago de Cali, calle 5 \# 62-00, Cali, Colombia, e-mail: jorge.silva04@usc.edu.co; ${ }^{3}$ Ingeniero Sanitario, Magíster en Ingeniería énfasis en Ingeniería Sanitaria y Ambiental. Escuela de Ingeniería de Recursos Naturales y del Ambiente-EIDENAR, Universidad del Valle, calle 13 \# 100-00, Cali, Colombia, e-mail: brayan.parra@correounivalle. edu.co; ${ }^{4}$ Ingeniera Sanitaria. Escuela de Ingeniería de Recursos Naturales y del Ambiente-EIDENAR, Universidad del Valle, calle 13 \# 100-00, Cali, Colombia, e-mail: viviana.ceron2@gmail.com; ${ }^{5}$ Ingeniero Sanitario, Magíster en Ingeniería Sanitaria, Candidato a Doctor en Ingeniería énfasis Ingeniería Sanitaria y Ambiental. Universidad del Valle, calle 13 \# 100-00, Cali, Colombia, e-mail: carlos.a.madera@correounivalle.edu.co
\end{abstract}

Rev. U.D.C.A Act. \& Div. Cient. 18(1): 69-79, Enero-Junio, 2015

\section{RESUMEN}

Los biosólidos tienen alto potencial de aprovechamiento en el sector agrícola, debido a su contenido de materia orgánica y de nutrientes. En esta investigación, se evaluó, durante un periodo de doce meses y en escala piloto, la influencia de la aplicación de biosólidos de una planta de tratamiento de aguas residuales sobre la calidad del suelo, las características morfológicas de la planta, la calidad del producto reflejada en grados brix, sacarosa y azúcares reductores y la productividad en términos de toneladas por hectárea, de un cultivo de caña de azúcar. El estudio, se realizó en una modalidad de bloques completos al azar con 3 tratamientos: suelo sin aplicación de nutrientes y fertilización mineral y con aplicación de biosólidos, con 3 réplicas por tratamiento. Se encontró que la aplicación de los biosólidos no alteró la calidad microbiológica y parasitológica del suelo, en cuanto a variables de interés sanitario. Las concentraciones de materia orgánica, nitrógeno mineral, fósforo y potasio, estuvieron dentro de los rangos típicos de los suelos del Valle del Cauca; las características morfológicas de la planta también se encontraron en el rango típico de la variedad empleada y en el rendimiento del cultivo respecto a sacarosa, grado brix y azúcares reductores. No se encontraron diferencias estadísticas significativas entre la fertilización mineral y la aplicación de los biosólidos, ratificando la viabilidad de este último en actividades agrícolas.

Palabras clave: Azúcares reductores, Biosólidos, Sacarosa, Saccharum officinarum, Suelo vertic endoaquepts.

\section{SUMMARY}

Biosolids have great utilization potential in the agricultural sector due to its content of organic matter and nutrients. This research evaluated over a period of twelve months and at pilot scale, the influence of the application of biosolids from one wastewater treatment plant on the soil quality, the morphological characteristics of the plant, the quality of product reflected in degrees brix, sucrose and reducing sugars and the crop yield in terms of tons per hectare of a sugarcane crop. The study was executed in a complete blocks modality at random form with 3 treatments: soil without nutrients application and mineral fertilization, and with biosolids application with 3 replications per treatment. It was found that the application of biosolids did not alter the microbiological and parasitological quality of the soil with regard to sanitary interest variables. Organic matter, mineral nitrogen, phosphorus and potassium concentrations, were found in the range of the soils of the Valle del Cauca; morphological features of 
the plant were found in the typical range of the variety used and the crop yield regarding sucrose, brix degrees and reducing sugars did not show significant statistical differences between the application of biosolids and mineral fertilization, thus ratifying the feasibility of the use of biosolids for agricultural application.

Key words: Biosolids, reducing sugars, Saccharum officinarum, sucrose, vertic endoaquepts soil.

\section{INTRODUCCIÓN}

La adecuada gestión de los subproductos generados en el tratamiento de aguas residuales es necesaria, para evitar que causen impactos negativos al ambiente o a la salud de la población. El lodo es el principal subproducto sólido y en el que se concentra gran parte de la contaminación química y microbiológica (Laturnus et al. 2007) y la digestión anaerobia es uno de los procesos de mayor aplicación para su estabilización, generando biosólidos.

Los biosólidos contienen materia orgánica y nutrientes, como nitrógeno, fósforo y potasio, en niveles que los hacen potencialmente aprovechables, como enmiendas orgánicas o fuente de nutrientes (Fuccz et al. 2007; Mo \& Zhang, 2012), en el sector agrícola. Estas prácticas son comúnmente empleadas en países en desarrollo, como Argentina, Brasil, Chile y México, de acuerdo a lo reportado por Torres et al. (2009); sin embargo, debido a que la digestión no garantiza la suficiente reducción de los patógenos (Silva et al. 2013a), la aplicación de los biosólidos se puede restringir a ciertos usos, de acuerdo con su clasificación, como lo establece la Norma 40 CFR parte 503 de la EPA (EPA, 1999; León et al. 2007).

La explotación agrícola de suelos en el país ha ido creciendo, causando un aumento en la demanda de mejoradores de suelos (DANE, 2012); adicionalmente, el incremento de Plantas de Tratamiento de Aguas Residuales-PTAR en las grandes ciudades, ha elevado la producción de biosólidos, encontrándose estudios que evalúan su aprovechamiento en cultivos de rábano (Quinchía \& Carmona, 2004; Silva et al. 2013b), mejoramiento de suelos de escombreras y de pastos (Ramírez \& Pérez, 2006; Sánchez et al. 2010) y de caña de azúcar (Silva et al. 2013a).

La caña de azúcar es un cultivo comercial de alta importancia para la economía nacional y, principalmente, para el Valle del Cauca, que ha dispuesto para este cultivo 224.000 hectáreas, que representan el $49 \%$ del área agrícola, logrando producir el $80 \%$ del azúcar que se consume en el país (ASOCAÑA, 2012a; 2012b). La demanda, tanto de agua para riego como de plaguicidas y fertilizantes, es alta (aproximadamente, $5 \mathrm{~kg} \mathrm{~N} ; 1,3 \mathrm{~kg} \mathrm{P}_{2} \mathrm{O}_{5} ; 6 \mathrm{~kg} \mathrm{~K} \mathrm{O}_{2} ; 0,9 \mathrm{~kg} \mathrm{Mg} \mathrm{y} \mathrm{0,4kg} \mathrm{S}$ por tonelada, cosechada en base seca) (Ciampitti \& García,
2007). Debido a que es un cultivo semiperenne que continúa en producción durante años, esta demanda es constante, siendo los impactos económicos y ambientales significativamente altos (Chaves, 1999; Pérez \& Álvarez, 2009).

El uso de fertilizantes con menores efectos sobre el ambiente y menores costos de producción y las experiencias mencionadas hacen que la utilización de materiales, como los biosólidos, que cumplen con los dos criterios; en consecuencia, permite su viabilidad como mejorador de suelos, con fines agrícolas (Jurado et al. 2012).

En esta investigación, se evaluó la influencia de la aplicación de los biosólidos generados en la PTAR Cañaveralejo, de la ciudad de Cali-PTAR-C, sobre la calidad de un suelo Vertic endoaquepts, cultivado con caña de azúcar, en términos de sus propiedades químicas, microbiológicas y parasitológicas, sobre las características morfológicas de la planta y la calidad del producto, tanto en términos de contenido de sacarosa, grados brix, azúcares reductores como de rendimiento del cultivo.

\section{MATERIALES Y MÉTODOS}

Características del suelo de cultivo y los biosólidos: $\mathrm{El}$ estudio, se llevó a cabo en las instalaciones de la PTAR-Cañaveralejo-PTAR-C de la ciudad de Cali, en un suelo Vertic endoaquepts, en zona agroecológica bosque seco tropical, con una topografía plana, altitud $967 \mathrm{msnm}$, temperatura promedio de $24^{\circ} \mathrm{C}$ y precipitación pluvial anual entre 1000 y $1500 \mathrm{~mm}$. Previo a la siembra, se efectuaron procesos de adecuación y de preparación de 0,5ha del terreno, con labores de rastrillado, subsolado y surcado a $1,65 \mathrm{~m}$.

Los biosólidos provenían de la línea de lodos: espesamiento, digestión anaerobia y deshidratación mecánica en filtros prensa de la PTAR-C, que opera bajo la modalidad de tratamiento primario avanzado-TPA. Las variables químicas evaluadas en el suelo, se midieron al inicio, a los 4, 10 y 12 meses; mientras que a los biosólidos, al inicio de la siembra. Los parámetros que se evaluaron fueron $\mathrm{pH}$, humedad, carbono orgánico$\mathrm{CO}$, nitrógeno total Kjeldahl-NTK, $\mathrm{N}$-amoniacal- $\mathrm{N}_{-}-\mathrm{NH}_{4}{ }^{+}, \mathrm{N}$ nitritos- $\mathrm{N}-\mathrm{NO}_{2}{ }^{-}$y $\mathrm{N}$-nitratos- $\mathrm{N}-\mathrm{NO}_{3}{ }^{-}$, fósforo total-P total, potasio$\mathrm{K}^{+}$, sodio- $\mathrm{Na}^{+}$, calcio- $\mathrm{Ca}^{+2}$ y magnesio- $\mathrm{Mg}^{+2}$, de acuerdo con los métodos de extracción Kjeldahl (1883) y USDA \& NRCS (2004); adicionalmente, se determinó el N-mineral en términos de $\mathrm{N}$-amoniacal, $\mathrm{N}$-nitritos y $\mathrm{N}$-nitratos, los cuales, forman el $\mathrm{N}$ disponible para plantas y microorganismos del suelo (Kjeldahl, 1883). Las variables microbiológicas y parasitológicas estudiadas fueron coliformes fecales, huevos de helmintos y Salmonella sp., de acuerdo con la EPA (1995).

Descripción del experimento: Se empleó un diseño experimental de bloques completos al azar, con tres tratamientos 
y tres repeticiones: suelo sin aplicación de nutrientes; fertilización química y aplicación de biosólido requerido por el cultivo. Cada tratamiento, se conformó con tres surcos de $20 \mathrm{~m}$ de largo x 1,5m de ancho y separación de 1,65m, entre surco.

El aporte de nutrientes para el cultivo en cada uno de los bloques, se calculó con base en las características fisicoquímicas iniciales del suelo y las necesidades del cultivo, para el proceso de fertilización. El tratamiento I correspondió al suelo sin ninguna aplicación de nutrientes; para el tratamiento II, se aplicó fertilizante mineral-FM, en una proporción 100:50:30 kg*ha ${ }^{-1}$ de $\mathrm{N}: \mathrm{P}_{2} \mathrm{O}_{5}: \mathrm{K}_{2} \mathrm{O}$, respectivamente $\mathrm{y}$, el tratamiento III, recibió una aplicación de biosólidos, de $41,78 \mathrm{t}^{*} \mathrm{ha}^{-1}$, considerado el aporte de nitrógeno del biosólido y su tasa de mineralización (Peñarete et al. 2013; Silva et al. 2013b).

La preparación del suelo consistió en dos pasadas de arado de discos y surcado. La FM, se realizó 30 días después de la germinación (Quintero, 1993) y los biosólidos fueron aplicados en el fondo del surco dos semanas antes de la siembra, para permitir la incorporación de los nutrientes al suelo. La semilla de caña de azúcar empleada fue la variedad CC-8592 - Cenicaña-Colombia, con aproximadamente $60 \mathrm{~cm}$ de longitud, distribuida dentro de cada surco y tapada con una capa de suelo, cerca de $10 \mathrm{~cm}$ y, el riego, se hizo de acuerdo con los requerimientos hídricos del cultivo, mediante aplicación por surcos con tubería de ventanas (Peñarete et al. 2013).

En cada riego, al agua de pozo se le evaluaron las variables químicas $\mathrm{pH}$, conductividad, $\mathrm{NTK}, \mathrm{N}-\mathrm{NH}_{4}{ }^{+}, \mathrm{N}_{-} \mathrm{NO}_{2}, \mathrm{~N}_{-} \mathrm{NO}_{3}{ }^{-}$, $\mathrm{P}_{\text {total }}, \mathrm{PO}_{4}{ }^{=}, \mathrm{K}^{+}, \mathrm{Na}^{+}, \mathrm{Ca}^{+2}, \mathrm{Mg}^{+2}, \mathrm{CO}_{3}{ }^{\circ}, \mathrm{HCO}_{3}{ }^{-}, \mathrm{Cl}^{-}, \mathrm{SO}_{4}{ }^{=}$y la relación de absorción de sodio-RAS (USDA \& NRCS, 2004; FAO, 2006); las variables microbiológicas y parasitológicas fueron las mismas caracterizadas al suelo y al biosólido. Para la evaluación de la respuesta morfológica del cultivo, se midieron la altura de la planta, con una frecuencia de 15 días y el diámetro y la distancia entre nodos, con una frecuencia de 30 días.

Culminados los 12 meses desde la siembra, se ejecutaron labores de corte y de recolección y se midieron el contenido de sacarosa, grados brix, azúcares reductores y toneladas de caña de azúcar por hectárea (Salgado et al. 2000). A partir de los resultados de sacarosa, se determinó el rendimiento del cultivo aplicando la ecuación 1.

$$
R=P_{s}-P_{p} \text { Ec. } 1
$$

Donde R es el rendimiento obtenido por el cultivo (\%); $\mathrm{Ps}_{\mathrm{s}}$ es el porcentaje de sacarosa obtenido (\%) y $\mathrm{P}_{\mathrm{p}}$ es el porcentaje de pérdidas (\%); para esto, se consideraron unas pérdidas cercanas al 1,5\%, para valores comerciales y para caña limpia, de acuerdo a Larrahondo \& Briceño (2004).

El valor teórico de azúcar o toneladas de azúcar por hectárea (TAH), se determinó utilizando la ecuación 2.

$$
\mathrm{TAH}=\mathrm{TCH}{ }^{*} \mathrm{R} \text { Ec. } 2
$$

Donde TAH son toneladas de azúcar por hectárea $\left(\mathrm{t}^{*}\right.$ ha $\left.\mathrm{t}^{-1}\right)$; TCH son las toneladas de caña de azúcar por hectárea ( $t / h a)$ y $\mathrm{R}$ es el rendimiento obtenido por el cultivo (\%).

Al final del estudio, se realizó un análisis de estadística descriptiva para las variables químicas, microbiológicas y parasitológicas del suelo, los biosólidos y el agua de pozo y para los parámetros que reflejan la respuesta morfológica del cultivo. Para las variables relacionadas con el rendimiento del cultivo, se efectuó un análisis de varianza con componente de submuestreo y un nivel de significancia del 95\%; las diferencias entre medias fueron determinadas utilizando el paquete estadístico de libre acceso "R" versión 2.15.0.

\section{RESULTADOS Y DISCUSIÓN}

La tabla 1 muestra la caracterización del suelo y el biosólido. El pH del suelo es alcalino y los contenidos de $\mathrm{N}, \mathrm{P}$ y K y bases intercambiables son de medios a altos y altos, respectivamente, características aptas para el cultivo de la caña de azúcar (Quintero, 1993); sin embargo, la relación Ca/Mg menor a la ideal, que corresponde a 3:1 y la relación $\mathrm{Mg} / \mathrm{K}$, superior a la ideal, que oscila entre 6 y 8 , pueden llevar a antagonismos, que afectan la absorción de calcio, de magnesio y de potasio, por parte de las plantas y, consecuentemente, el rendimiento del cultivo de caña de azúcar (Castro \& Gómez, 2010).

Las características de los biosólidos son consistentes con este tipo de materiales (Potisek et al. 2010) y su contenido de $\mathrm{MO}, \mathrm{N}_{-} \mathrm{NH}_{4}{ }^{+}$y P permiten catalogarlo como posible acondicionador o mejorador de las propiedades físicas, químicas e hidrodinámicas del suelo (Sousa et al. 2011; Peñarete et al. 2013); no obstante, la baja concentración de K puede ser limitante, ya que es esencial en la creación de pared celular, asimilación de carbono y la fotosíntesis y, en el caso de la caña de azúcar, se requiere en grandes cantidades, ya que de ello depende la cantidad de sacarosa que genere la planta (Chaves, 1999; Bolio et al. 2008). Desde el punto de vista del contenido de coliformes fecales, no se clasifica en las Clases A o B, según la Norma 40 CFR parte 503 de la EPA, por tanto, existen posibles restricciones en cuanto a su aplicación.

La tabla 2 presenta los resultados de caracterización de seis muestras de agua de pozo evaluadas durante el periodo de duración del cultivo. De acuerdo con USDA (1954), el agua 
Tabla 1. Resultados caracterización inicial suelo y biosólido.

\begin{tabular}{|c|c|c|c|}
\hline Variable & Unidad & Suelo & Biosólido PTAR-C \\
\hline \multicolumn{4}{|c|}{ Fisicoquímicas } \\
\hline $\mathrm{pH}$ & Unidades & 7,57 & 6,86 \\
\hline Humedad & $\%$ & - & 72,4 \\
\hline Materia Orgánica-MO & $\%$ & 4,47 & 15,52 \\
\hline NTK & $\%$ & - & 0,87 \\
\hline $\mathrm{N}$ amoniacal $-\mathrm{N}-\mathrm{NH}_{4}{ }^{+}$ & $\mathrm{mg}^{*} \mathrm{~kg}^{-1}$ & 1,89 & 2149,81 \\
\hline Nitratos-N-NO ${ }_{3}^{-}$ & $\mathrm{mg}^{*} \mathrm{~kg}^{-1}$ & 0,47 & 2,71 \\
\hline Nitritos-N-NO${ }_{2}^{-}$ & $\mathrm{mg}^{*} \mathrm{~kg}^{-1}$ & 0,0 & 1,74 \\
\hline Fósforo Total & $\mathrm{mg}^{*} \mathrm{~kg}^{-1}$ & 47,32 & 357,78 \\
\hline Potasio-K $\mathrm{K}^{+}$ & $\mathrm{cmol}^{*} \mathrm{~kg}^{-1}$ & 0,52 & 1,02 \\
\hline Sodio-Na ${ }^{+}$ & $\mathrm{cmol}^{*} \mathrm{~kg}^{-1}$ & 0,16 & 0,93 \\
\hline Calcio-Ca ${ }^{+2}$ & $\mathrm{cmol}^{*} \mathrm{~kg}^{-1}$ & 19,41 & 44,58 \\
\hline Magnesio-Mg ${ }^{+2}$ & $\mathrm{cmol}^{*} \mathrm{~kg}^{-1}$ & 7,36 & 4,77 \\
\hline \multicolumn{4}{|c|}{ Microbiológicas y Parasitológicas } \\
\hline Coliformes Fecales & UFC $* g^{-1}$ & 0,0 & $2,20 \times 10^{8}$ \\
\hline Huevos de Helmintos & $\mathrm{HH}^{*} \mathrm{~g}^{-1}$ & 0,0 & 7,0 \\
\hline Salmonella sp & Presencia/Ausencia & Ausencia & Ausencia \\
\hline
\end{tabular}

del pozo se clasifica como C2-S1, de salinidad media, apta para riego de plantas moderadamente sensibles a la salinidad y un bajo contenido de sodio; la mayoría de los parámetros evaluados, se encuentran dentro de los límites establecidos por FAO (1985), pudiéndose emplear para riego, pero con precauciones, como realizar lavados frecuentes, cuyo fin es evitar la acumulación de las sales en el suelo.

La tabla 3 presenta los resultados de caracterización fisicoquímica y microbiológica del suelo durante los meses 0,4 , 10 y 12 del cultivo, en cada uno de los tratamientos. Se observa que los biosólidos aportaron materia orgánica, nitrógeno, fósforo y potasio, siendo más significativo el aporte de nitrógeno amoniacal y fósforo, mientras que la fertilización mineral aportó, fundamentalmente, nitrógeno y potasio, debido a la aplicación específica de urea y cloruro de potasio.

En general, los valores de materia orgánica-MO encontrados en los tratamientos son cercanos a los típicos en suelos del valle geográfico del río Cauca, que oscilan entre 2 y $4 \%$ (Quintero, 1995). El N es el nutriente que más limita la producción especializada de caña de azúcar (CENICAÑA, 2002; Yang et al. 2011), lo que hace de la fertilización una práctica habitual, siendo los requerimientos variables, de acuerdo a la variedad a sembrar y al tipo de suelo; en este caso, el biosólido proporcionó el $\mathrm{N}$ suficiente para el desarrollo de la planta, pero no lo suficiente para lograr mayores resulta- dos, en cuanto al rendimiento del cultivo. Respecto al K que presentó niveles importantes en el Tratamiento II, se observa una importante reducción en los primeros meses, debido a su uso por la planta para la creación de pared celular, asimilación de carbono y la fotosíntesis; según CENICAÑA (2002), la variedad CC85-92 extrae grandes cantidades de $\mathrm{K}$ del suelo.

El contenido de microorganismos en el suelo, como se presenta en la tabla 3, indica presencia de coliformes fecales en los tres tratamientos, para el mes inicial y posterior a la aplicación del biosólido. Para los siguientes meses, el valor fue cero, debido, probablemente, a factores ambientales, como la temperatura, la radiación solar y la humedad (Fuccz et al. 2007). Asimismo, la precipitación también influye directamente sobre la supervivencia de microorganismos de suelos tratados con biosólidos (Zaleski et al. 2005).

La presencia de huevos de Helmintos fue detectable en el mes 0 , con valores no superiores a $3 \mathrm{HH}^{*} \mathrm{~g}^{-1}$, observándose su eliminación en los meses posteriores. Para el caso de la Salmonella sp., fue ausente en el biosólido y el suelo; según Smith (2009), la supervivencia en el suelo de Salmonella sp., comúnmente, es de alrededor de dos meses, debido a la variedad de microorganismos que pueden habitar en el mismo, en especial los heterótrofos. 
Tabla 2. Resultados de caracterización del agua de pozo.

\begin{tabular}{|c|c|c|c|c|}
\hline Variables & Unidades & Valor $^{p}$ & Deviación Estándar & Valores FAO* \\
\hline \multicolumn{5}{|c|}{ Químicas } \\
\hline $\mathrm{pH}$ & Unidades & $6,5 * * *$ & - & - \\
\hline NTK & $\mathrm{mg} \mathrm{N} \mathrm{N}^{-1}$ & 3,77 & 3,63 & $0-10$ \\
\hline N. amoniacal- $\mathrm{N}-\mathrm{NH}_{4}{ }^{+}$ & $\mathrm{mg} \mathrm{NH}_{3} * \mathrm{~L}^{-1}$ & 2,44 & 1,81 & $0-5$ \\
\hline Nitratos-N-NO 3 & $\mathrm{mg} \mathrm{NO}_{3} * \mathrm{~L}^{-1}$ & 12,26 & 14,03 & - \\
\hline Nitritos-N-NO 2 & $\mathrm{mg} \mathrm{NO}{ }_{2} * \mathrm{~L}^{-1}$ & 1,63 & 2,52 & - \\
\hline Fósforo total & $\mathrm{mg} \mathrm{P}^{*} \mathrm{~L}^{-1}$ & 1,04 & 1,15 & $0-2$ \\
\hline Fosfatos & $\mathrm{mg} \mathrm{PO} * \mathrm{~L}^{-1}$ & 0,46 & 0,87 & $0-2$ \\
\hline $\begin{array}{l}\text { Conductividad } \\
\text { eléctrica }\end{array}$ & $\mathrm{mS}^{*} \mathrm{~cm}^{-1}$ & 0,45 & 0,11 & $0-3$ \\
\hline Potasio- $\mathrm{K}^{+}$ & $\mathrm{mg}^{*} \mathrm{~L}^{-1}$ & 6,73 & 0,32 & $0-2$ \\
\hline Sodio-Na ${ }^{+}$ & $m g^{*} \mathrm{~L}^{-1}$ & 54,58 & 2,63 & $0-920$ \\
\hline Calcio-Ca $^{+2}$ & $\mathrm{mg} * \mathrm{~L}^{-1}$ & 37,64 & 4,57 & $0-400$ \\
\hline Magnesio-Mg ${ }^{+2}$ & $\mathrm{mg} * \mathrm{~L}^{-1}$ & 11,13 & 1,49 & $0-60,75$ \\
\hline Total Cationes & $\mathrm{mg}^{*} \mathrm{~L}^{-1}$ & 58,74 & 18,29 & \\
\hline Carbonato- $\mathrm{CO}_{3}=$ & $\mathrm{mg} * \mathrm{~L}^{-1}$ & ND* & ND* & $0-30$ \\
\hline Bicarbonato- $\mathrm{HCO}_{3}{ }^{-}$ & $\mathrm{mg} * \mathrm{~L}^{-1}$ & 217,84 & 55,41 & $0-610$ \\
\hline Cloruro- $\mathrm{Cl}^{-}$ & $\mathrm{mg}^{*} \mathrm{~L}^{-1}$ & 43,25 & 25,24 & $0-1064$ \\
\hline Sulfato- $\mathrm{SO}_{4}=$ & $\mathrm{mg} * \mathrm{~L}^{-1}$ & 39,86 & 44,19 & $0-960,5$ \\
\hline Total Aniones & $\mathrm{mg} * \mathrm{~L}^{-1}$ & 237,10 & 287,51 & - \\
\hline RAS** & - & 2,20 & 0,02 & - \\
\hline \multicolumn{5}{|c|}{ Microbiológicas y Parasitológicas } \\
\hline Coliformes Fecales & $\mathrm{UFC}^{*} 100 \mathrm{~mL}^{-1}$ & $1,33 \times 10^{2}$ & $2,80 \times 10^{2}$ & - \\
\hline Huevos de Helmintos & $\mathrm{UFC}^{*} 100 \mathrm{~mL}^{-1}$ & 0 & 0 & - \\
\hline Salmonella sp. & Presencia/Ausencia & Ausente & Ausente & - \\
\hline
\end{tabular}

*N.D: No Detectado, **RAS-Relación Adsorción Sodio, ***Medina, p: Promedio.

La figura 1 muestra las características morfológicas del cultivo, la distancia entre nodos, que varió entre $12-13 \mathrm{~cm}$, el diámetro entre 30,5 - 31,6mm y la altura de la planta entre $305-325 \mathrm{~cm}$ para los tratamientos, resultados que no difieren de los reportados para la variedad CC85-92: distancia entre nodos entre $13-16 \mathrm{~cm}$ y diámetro entre $30-32 \mathrm{~mm}$ (CENICAÑA, 2003) y altura de la planta entre 292 y $315 \mathrm{~cm}$ (CENICAÑA, 2010).
La aplicación del tratamiento con biosólidos, en comparación con el testigo y la fertilización mineral, indican que son plantas de menor tamaño, con diámetros promedios y mayor distancia entre nodos; no obstante, dichos valores no se alejan de los valores típicos morfológicos de la variedad CC85-92 (CENICAÑA, 2003), lo cual, indica que la aplicación de biosólidos, como tratamiento, no causa un efecto negativo sobre el desarrollo de la planta. 
Tabla 3. Resultados de caracterización del suelo.

\begin{tabular}{|c|c|c|c|c|c|c|}
\hline \multicolumn{7}{|c|}{ Caracterización fisicoquímica } \\
\hline Tratamiento & Parámetro & Unidad & Mes 0 & Mes 4 & Mes 10 & Mes 12 \\
\hline Tratamiento I & \multirow{3}{*}{ Materia Orgánica } & \multirow{3}{*}{$\%$} & 4,47 & 4,72 & 3,79 & 4,0 \\
\hline Tratamiento II & & & 4,2 & 4,52 & 3,85 & 4,4 \\
\hline Tratamiento III & & & 4,81 & 4,4 & 4,42 & 4,11 \\
\hline Tratamiento I & \multirow{3}{*}{ N. mineral* } & \multirow{3}{*}{$\mathrm{mg}^{*} \mathrm{~kg}^{-1}$} & 2,36 & 16,73 & 35,45 & 15,84 \\
\hline Tratamiento II & & & 1,4 & 168,59 & 53,65 & 4,9 \\
\hline Tratamiento III & & & 104,2 & 23,73 & 30,01 & 2,97 \\
\hline Tratamiento I & \multirow{3}{*}{ P-Fósforo total } & \multirow{3}{*}{$\mathrm{mg}^{*} \mathrm{~kg}^{-1}$} & 47,32 & 53,94 & 48,78 & 47,03 \\
\hline Tratamiento II & & & 44,38 & 57,94 & 51,72 & 60,05 \\
\hline Tratamiento III & & & 183,31 & 60,79 & 38,71 & 62,88 \\
\hline Tratamiento I & \multirow{3}{*}{ K-Potasio } & \multirow{3}{*}{$\mathrm{cmol}^{*} \mathrm{~kg}^{-1}$} & 0,52 & 0,68 & 0,53 & 0,62 \\
\hline Tratamiento II & & & 0,46 & 2,07 & 0,54 & 0,61 \\
\hline Tratamiento III & & & 0,6 & 0,69 & 0,55 & 0,6 \\
\hline \multicolumn{7}{|c|}{ Caracterización microbiológica } \\
\hline \multirow{3}{*}{ Tratamiento I } & Coliformes Fecales & UFC* $g^{-1}$ & $1,57 \times 10^{6}$ & 0,00 & 0,00 & 0,00 \\
\hline & Huevos de Helmintos & $\mathrm{HH}^{*} \mathrm{~g}^{-1}$ & 1,00 & 0,00 & 0,00 & 0,00 \\
\hline & Salmonella sp. & $\begin{array}{c}\text { Presencia/ } \\
\text { Ausencia }\end{array}$ & Ausente & Ausente & Ausente & Ausente \\
\hline \multirow{3}{*}{ Tratamiento II } & Coliformes Fecales & $\mathrm{UFC}^{*} \mathrm{~g}^{-1}$ & $1,50 \times 10^{6}$ & 0,00 & 0,00 & 0,00 \\
\hline & Huevos de Helmintos & $\mathrm{HH}^{*} \mathrm{~g}^{-1}$ & 3,00 & 0,00 & 0,00 & 0,00 \\
\hline & Salmonella sp. & $\begin{array}{l}\text { Presencia/ } \\
\text { Ausencia }\end{array}$ & Ausente & Ausente & Ausente & Ausente \\
\hline \multirow{3}{*}{ Tratamiento III } & Coliformes Fecales & $\mathrm{UFC}^{*} \mathrm{~g}^{-1}$ & $3,33 \times 10^{6}$ & 0,00 & 0,00 & 0,00 \\
\hline & Huevos de Helmintos & $\mathrm{HH}^{*} \mathrm{~g}^{-1}$ & 2,00 & 1,00 & 0,00 & 0,00 \\
\hline & Salmonella sp. & $\begin{array}{c}\text { Presencia/ } \\
\text { Ausencia }\end{array}$ & Ausente & Ausente & Ausente & Ausente \\
\hline
\end{tabular}

*N. mineral: N. amoniacal-N-NH${ }_{4}{ }^{+}+$Nitritos- $-\mathrm{NO}_{2}{ }^{-}+$Nitratos- $-\mathrm{N}^{-} \mathrm{NO}_{3}{ }^{-}$.

La tabla 4 muestra los resultados de la medición de los niveles de sacarosa, grados brix, azúcares reductores y toneladas de caña de azúcar por hectárea $\left(\mathrm{t}^{*} \mathrm{ha}^{-1}\right)$, al final del periodo del cultivo. En esta tabla, se observa que la aplicación de los biosólidos presentó resultados superiores al promedio reportado por los ingenios azucareros del país, para las variables sacarosa grados brix y toneladas de caña por hectárea, que corresponden a $12,92 \%, 16 \%$ y $114,6 \mathrm{t}^{*} \mathrm{ha}^{-1}$, respectivamente (CENICAÑA, 2010); los azúcares reductores se encontraron en el rango reportado por los ingenios entre 1 a $5 \%$ (CENICAÑA, 2002). Es probable que la baja concentración de $\mathrm{K}$ en los biosólidos haya sido uno de los factores por el cual el Tratamiento III no logró resultados significativos, en cuanto a producción de $\mathrm{TCH}$, comparados con el Tratamiento II, en que se hizo fertilización mineral, ya que este nutriente es fun- damental para el desarrollo de la planta, lo que incide sobre la cantidad de masa de la caña de azúcar.

En cuanto al rendimiento del cultivo y las TAH fueron de 15,$97 ; 15,52$ y $16,79 \%$ y 20,$67 ; 22,30$ y $20,36 t^{*}$ ha $^{-1}$, para el testigo, la fertilización mineral y el biosólido, respectivamente; algunos de estos valores difieren de lo reportado por CENICAÑA (2010), debido, probablemente, a prácticas agrícolas de los ingenios, como acondicionamiento de suelos, de edad de corte, de aplicaciones excesivas de nitrógeno, entre otros (Larrahondo \& Briceño, 2004).

Aunque el análisis de varianza para un nivel de significancia del 95\% $(p<0,05)$ no presentó diferencias estadísticas entre tratamientos en términos de sacarosa, grados brix y 

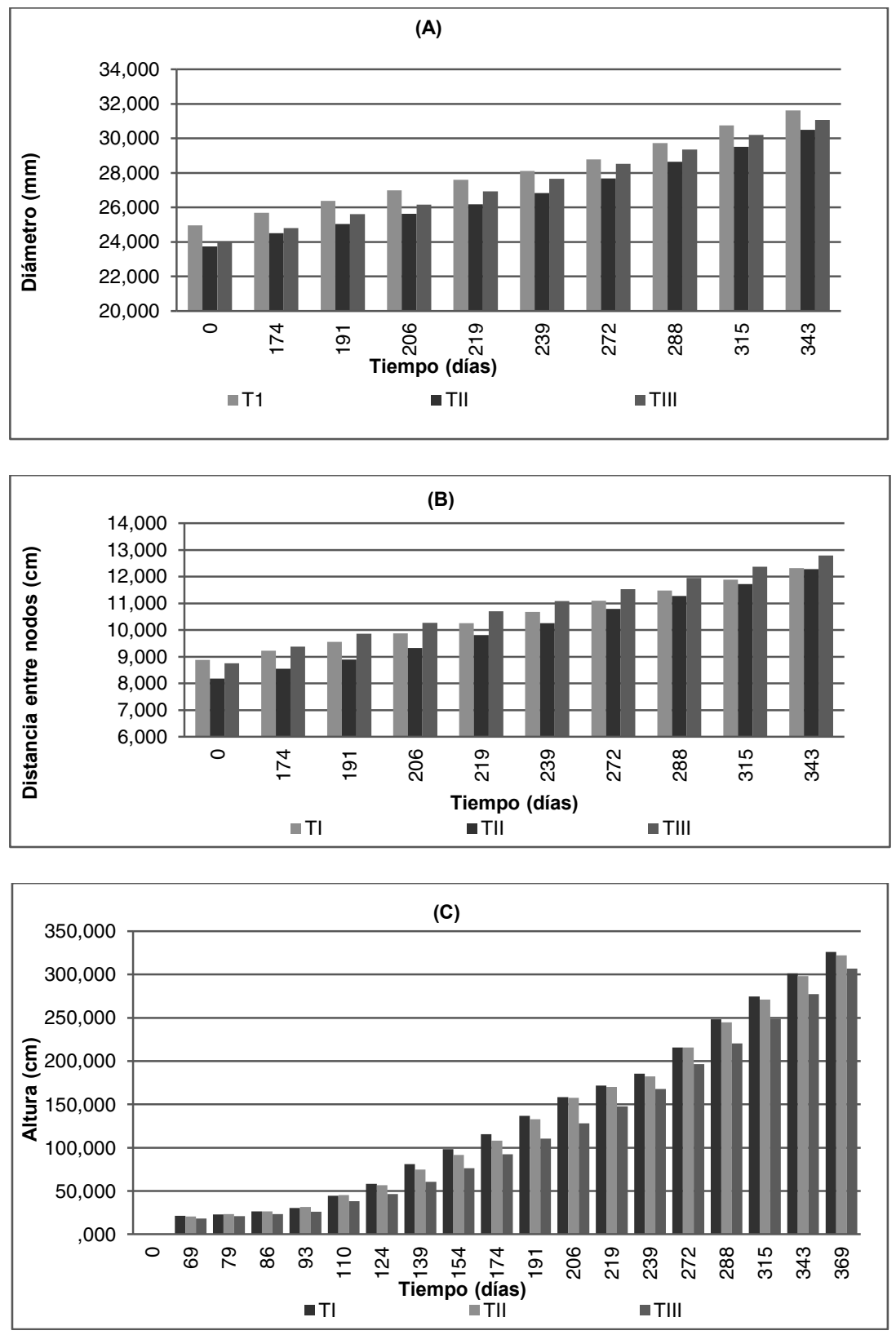

Figura 1. Diámetro, distancia entre nodos y altura de la planta durante el tiempo del cultivo.

azúcares, sí lo evidenció para la TCH; al emplear la prueba de Tukey, se encontró una diferencia entre los tratamientos II y III, de 20,6 TCH. En general, los resultados encontrados son similares a los encontrados por Armelin et al. (2010) y Franco et al. (2010), quienes concluyeron que al aplicar biosólidos y fertilizante en un cultivo de caña de azúcar, no se encontraron diferencias estadísticas.
La fertilización mineral generó la mayor producción de caña en cuanto a $\mathrm{t}^{*} \mathrm{ha}^{-1}$, similar a los resultados de Marques et al. (2007), quienes aplicaron entre 30 y 40t/ha de biosólidos, provenientes de una PTAR de la ciudad de São Paulo-Brasil, un cultivo de caña de azúcar. A nivel nacional, Silva et al. (2013a) registraron que dosis entre 71,6 y 163,5t*ha ${ }^{-1}$ pueden suplir el requerimiento de $\mathrm{N}$ del cultivo de caña de azúcar, pero dosis 
Tabla 4. Análisis exploratorio para las variables respuesta por tratamiento.

\begin{tabular}{|c|c|c|c|c|c|c|c|c|c|c|c|c|c|}
\hline \multirow{2}{*}{$\mathrm{X}$} & \multirow{2}{*}{$\mathbf{P}$} & \multicolumn{3}{|c|}{ \% Sacarosa } & \multicolumn{3}{|c|}{ \%Grados Brix } & \multicolumn{4}{|c|}{ \% Azucares Reductores } & \multicolumn{2}{|c|}{$\mathrm{TCH}(\mathrm{t} / \mathrm{ha})$} \\
\hline & & TI & TII & TIII & TI & TII & TIII & TI & TII & TIII & TI & TII & TIII \\
\hline \multirow{5}{*}{9} & Min. & 15,37 & 15,37 & 17,11 & 15,7 & 16,8 & 17,5 & 0,42 & 0,65 & 0,43 & 123,30 & 139,10 & 106,70 \\
\hline & Máx. & 18,59 & 18,56 & 19,2 & 19,1 & 17,4 & 18,8 & 1,79 & 1,64 & 1,82 & 137,90 & 147,20 & 139,80 \\
\hline & Media & 17,47 & 17,02 & 18,29 & 17,3 & 17,2 & 18,2 & 1,04 & 1,07 & 1,04 & 129,40 & 143,7 & 123,00 \\
\hline & $\begin{array}{l}\text { Desv. } \\
\text { Esta }\end{array}$ & 1,82 & 1,59 & 1,07 & 1,7 & 0,34 & 0,65 & 0,69 & 0,51 & 0,71 & 7,57 & 4,15 & 16,55 \\
\hline & CV (\%) & 10,42 & 9,34 & 5,85 & 9,83 & 1,98 & 3,58 & 66,35 & 47,7 & 68,27 & 5,85 & 2,89 & 13,45 \\
\hline
\end{tabular}

X-Número de muestra, P-Parámetro, TI-Tratamiento I, TII-Tratamiento II, TIII-Tratamiento III, TCH- toneladas de caña de azúcar por hectárea.

superiores, pueden incrementar la pérdida de $\mathrm{N}$ y convertirse en una fuente de contaminación de los acuíferos.

Autores, como Gardner et al. (2010) y Sousa et al. (2011) afirman que la utilización de biosólidos en la agricultura acarrea beneficios económicos y principalmente ambientales, pues ayuda a la disminución de gases de efecto invernaderoGEI, por la no aplicación de fertilizantes minerales, induce al aumento en la diversidad microbiológica de los suelos y facilita la asimilación de nutrientes por parte del cultivo y estimula el re-uso de recursos, como el $\mathrm{N}$ y el $\mathrm{P}$, lo que contribuye al flujo equilibrado de nutrientes en los ecosistemas.

Aunque para el sector azucarero, en especial los proveedores de caña de azúcar, es de gran importancia obtener mayores resultados en cuanto a TCH, estudios realizados por Luna et al. (1995), Luna (2006) y Salles et al. (2008), aseveran que para los ingenios azucareros ha tomado mayor relevancia el contenido de sacarosa, grados brix y azúcares reductores presentes en la caña, pues de ello depende la cantidad de azúcar producida.

En general, esta investigación permite concluir que el suelo presentó contenidos adecuados de materia orgánica y fósforo y una relación Ca:Mg de 2:1; sin embargo, fue necesario aplicar nitrógeno y potasio, para garantizar los requerimientos y el desarrollo esperado del cultivo. La disponibilidad de materia orgánica y macronutrientes del biosólido, indica que tiene el potencial de ser aprovechado y suplir los requerimientos del cultivo de la caña de azúcar, en términos de $\mathrm{Ny}$ $\mathrm{P}$; a pesar de la presencia de coliformes fecales y huevos de helmintos en los biosólidos, que podrían representar restricciones para su aplicación, disminuyen durante el tiempo del cultivo, lo que implica que los riesgos a los que se somete el operario del campo son bajos.

Por otro lado, la dosis de $41,78 \mathrm{t} * \mathrm{ha}^{-1}$ de biosólidos proporcionó los nutrientes necesarios para el desarrollo, el crecimiento y el rendimiento del cultivo, con valores similares a los obtenidos con la dosis de fertilización mineral de $39,1 \mathrm{~kg}^{*}$ ha ${ }^{-1}$.

En este estudio, se evidenció que las características morfológicas del cultivo, como altura, diámetro y distancia entre nodos, no mostraron diferencias estadísticas entre los tratamientos, alcanzándose, en todos los casos, valores dentro de los rangos esperados para la variedad CC85-92, evidenciándose que la aplicación de biosólidos no incide de manera negativa sobre el desarrollo.

Aunque el rendimiento relacionado al $\mathrm{TCH}$ del tratamiento con fertilización mineral fue superior estadísticamente, para los ingenios azucareros ha tomado mayor relevancia el contenido de sacarosa, grados brix y azúcares reductores, pues están asociados a la cantidad de azúcar producida; en el estudio, no se hallaron diferencias estadísticas entre los tratamientos y, en el caso del tratamiento con biosólidos, los valores superaron el promedio reportado por los ingenios azucareros del país.

Finalmente, para cada tipo de cultivo y de suelo, es recomendable definir la dosis óptima de biosólido a aplicar, con el fin de obtener los mejores indicadores de productividad del cultivo evaluado.

Agradecimientos: Los autores agradecen a EMCALI E.I.C.E E.S.P por permitir realizar la investigación en la PTAR-C, a Colciencias y la Universidad del Valle, por el apoyo técnico y financiero en el proyecto código 1106-489-25147 y por la financiación del estudiante Jorge Silva, como becario de doctorado nacional. Conflicto de interés: Certificamos la originalidad y el carácter inédito del manuscrito elaborado y revisado con la participación de todos los autores, quienes declaramos que no existe conflicto de intereses que ponga en riesgo la validez de los resultados. 


\section{BIBLIOGRAFÍA}

1. ARMELIN, M.; ABREU, C.; CHATARINO, M.; SAIKI, M.; RIBEIRO, A.; FRANCO, A.; FERNANDES, H. 2010. Determinação de antimônio, arsênio, cádmio e tório em amostras de caldo de cana cultivada em solo tratado com lodo de esgoto. J. Braz. Soc. Ecotoxicol. 5(1):8-84.

2. ASOCAÑA. 2012a. Análisis Estructural 2004-2005. Cali-Colombia. ASOCAÑA.Disponible desde Internet en: http://www.asocana.org/StaticContentFull. aspx?SCid=19\#top (con acceso el 01/03/2012).

3. ASOCAÑA. 2012b. Informe Anual ASOCAÑA 20112012. Asociación de cultivadores de caña de azúcar de colombia. Cali-Colombia. 128p.

4. BOLIO, L.; SALGADO, G.; PALMA, L.; DEL CARMEN, L.; CASTELÁN, E.; ETCHEVERS, B. 2008. Dinámica del potasio en vertisoles y fluvisoles cultivados con caña de azúcar. Terra Latinoamericana 26(3): 253263.

5. CASTRO, H.; GÓMEZ, M. 2010. Fertilidad de suelos y Fertilizantes. En: Burbano, H.; Mojica, F. (eds.). Ciencia del Suelo. Principios básicos. Sociedad Colombiana de la Ciencia del Suelo. Bogotá, Colombia.. p.213-303.

6. CENICAÑA. Centro de Investigación de la Caña 2002. Características agronómicas y de productividad de la Variedad Cenicaña Colombia (CC) 85-92. Centro de Investigación de la Caña de Azúcar de Colombia. Cali. 80p.

7. CENICAÑA. Centro de Investigación de la Caña. 2003. Catálogo de Variedades: Segunda edición. Serie Técnica No 31. 34p.

8. CENICAÑA. Centro de Investigación de la Caña. 2010. Informe Anual 2010. CENICAÑA.Centro de Investigación de la Caña de Azúcar de Colombia. Cali. 166p.

9. CIAMPITTI, I.; GARCÍA, F. 2007. Archivo Agrónimico. Requerimiento nutricionales. Absorción y extracción de macronutrientes y nutrientes secundarios. International Plant Nutrition Institute. Buenos Aires. p.1-4.

10. CHAVES, S. 1999. El Nitrógeno, Fósforo y Potasio en la Caña de Azúcar. DIECA. Liga Agrícola Industrial de la Caña de Azúcar. Dirección de Investigación y Extensión de la Caña de Azúcar -DIECA. San José.129p.
11. DANE. 2012. Producto Interno Bruto - Tercer Trimestre de 2012. DANE.Departamento Nacional de Estadística. Bogotá D.C Colombia. 18p.

12. EPA. 1995. Process Design Manual, Land Application of Sewage Sludge and Domestic Septage. EPA. United States Environmental Protection Agency. Washington D.C. 302p.

13. EPA. 1999. Biosolids Generation, Use, and Disposal in The United States. EPAEnvironmental Protection Agency. 74p.

14. FAO. 1985. Water quality for agriculture. FAO.Food and Agriculture Organization of the United Nations. Rome. 29p.

15. FAO. 2006. Evapotranspiración del cultivo. Guías para la determinación de los requerimientos de agua de los cultivos FAO. Organización de las Naciones Unidas para la Alimentación y la Agricultura. Roma. 299p.

16. FRANCO, A.; HAMILTON, A.; PERECIN, D.; CARVALHO, O.; RIBEIRO, G.; SANTORO, B. 2010. Sewage sludge as nitrogen and phosphorus source for caneplant and first ratoon crops. Rev. Bras. Ciência Solo 34(2):553-561.

17. FUCCZ, G.; GÓMEZ, M.; CÁRDENAS, G.M.; CAMPOS, P.C. 2007. Comportamiento de coliformes fecales como indicadores bacterianos de contaminación fecal en diferentes mezclas de biosólido y estériles utilizados para la restauración ecológica de la cantera soratama, Bogotá. Univers. Scient. 12:111-120.

18. GARDNER, W.; BROERSMA, K.; NAETH, A.; CHANASYK, D.; JOBSON, A. 2010. Influence of biosolids and fertilizer amendments on physical, chemical and microbiological properties of copper mine tailings. Can. J. Soil. Sci. 90:571-583.

19. JURADO, G.P.; LUNA, L.M.; BARRETERO, H. 2012. Aprovechamiento de biosólidos como abonos orgánicos en pastizales áridos y semiáridos. Rev. Mex. Cienc. Pec. 42(3):379-395.

20. KJELDAHL, J. 1883. Neue Methode zur Bestimmung des Stickstoffs in organischen Körpern. Z. Anal. Chem. 22:366-382.

21. LARRAHONDO A., J.; BRICEÑO, B. 2004. Una Aproximación a la reducción de las pérdidas de sacarosa entre cosecha y molienda en el sector azucarero colombiano. CENICAÑA Serie Procesos Industriales No 3. 22p. 
22. LATURNUS, F.; VON ARNOLD, K.; GRON, C. 2007. Organic contaminants from sewage sludge applied to agricultural soils.false alarm regarding possible problems for food safety? Env. Sci. Pollut Res. 14(1):5360.

23. LEÓN, Z.; TREJOS, R.; CÁRDENAS, G.; CAMPOS, P. 2007. Comportamiento de los fagos somáticos en mezclas de biosólido y áridos utilizados para la restauración ecológica de la Cantera Soramata, Localidad de Usaquén, Bogotá. Univer. Scient. 12(2):99109.

24. LUNA, G.; COCK, J.; PALMA, A.; DÍAZ, L.; MORENO, C. 1995. Análisis de la productividad en la agroindustria azucarera de Colombia y perspectivas para aumentarla. Cali, CENICAÑA. p.373-394.

25. LUNA, G. 2006. Aumento de la productividad de caña de azúcar por unidad de área cultivada. Tecnicaña. 10(8):4-19.

26. MARQUES, M.; BELLINGIERRI, P.; MARQUES, T.; NOGUEIRA, T. 2007. Qualidade e produtividade de canade-açúcar cultivada em solo com doses crescentes de lodo de esgoto. Biosci. J. 23:111-122.

27. MO, W.; ZHANG, O. 2012. Can Municipal Wastewater systems be carbon netrual? J. Environm. Manag. 112:360-367.

28. PEÑARETE, W.; SILVAL, L.J.; URRUTIA, N.; DAZA, M; TORRES, L.P. 2013. Effect of biosolids application on soil physical properties of a sugarcane crop. Acta Agron. 62(3):251-260.

29. PÉREZ, R.; ÁLVAREZ, R. 2009. Deuda social y ambiental del negocio de la caña de azúcar en Colombia. Responsabilidad social empresarial y subsidios implícitos en la industria cañera. Análisis en el contexto del conflicto corteros-empresarios. R. F. Ed. ARFO (Colombia). 70p.

30. POTISEK, M.; FIGUEROA, U.; GONZÁLEZ, G.; JASSO, R.; ORONA, I. 2010. Aplicación de biosólidos al suelo y su efecto sobre contenido de materia orgánica y nutrimentos. Soc. Mex. Ciencia Suelo, A.C. Terra Latinoam. 28(4):327-333.

31. QUINCHÍA, A.; CARMONA, D. 2004. Factibilidad de disposición de los biosólidos generados en una planta de tratamiento de aguas residuales combinada. Rev. EIA. 2:89-108.
32. QUINTERO, D. 1993. Interpretación del análisis de suelo y recomendaciones de fertilizantes para la caña de azúcar. Cenicaña serie técnica. No. 14. 12p.

33. QUINTERO, D. 1995. Fertilización y Nutrición. El Cultivo de la caña en la zona azucarera de Colombia. Cali, CENICAÑA. p.153-157.

34. RAMÍREZ, P.; PÉREZ, A. 2006. Evaluación del potencial de los biosólidos procedentes del tratamiento de aguas residuales para uso agrícola y su efecto. Rev. Fac. Nal. Agr. Medellín. 59(2):3543-3556.

35. SALGADO, G.S.; NÚÑ̃EZ E., J.; PEÑA, J.; ETCHEVERS, D.; SOTO, R. 2000. Respuesta de la soca de caña de azúcar a la fertilización NPK. Agroc. 34:689-698.

36. SALLES, S.; PLÀ, A.; GOMES, F. 2008. La Optimización del Cultivo de Variedades de Caña de Azúcar. Rev. Inv. Operac. 29(1):26-33.

37. SÁNCHEZ, I.; CIFUENTES, D.; ANTE, C. 2010. Seguimiento a patógenos presentes en biosólido empleado como enmienda para revegetalizar un talud. Rev. Ingenierías. U. Medellin. 17:29-40.

38. SILVA, J.; TORRES, P.; MOSQUERA, J. 2013a. Evaluación de la mineralización de biosólidos de plantas de tratamiento de aguas residuales domésticas. $\mathrm{R}$. Bras. Eng. Agríc. Amb.l 17(4):434-442.

39. SILVA, L.J.A.; BEDOYA, R.D.; TORRES, L.P. 2013b. Evaluación del potencial de aplicación de biosólidos higienizados en el cultivo de rábano. Acta Agron. 62(2):155-164.

40. SMITH, S. 2009. Risks Associated with Biosolids Reuse in Agriculture. Jímenez, B.; Rose, J. (eds.). Urban Water Security: Managing Risks. Unesco-IHP Series. 303p.

41. SOUSA, G.; FANGUEIRO, D.; DUARTE, E.; VASCONCELOS, E. 2011. Reuse of treated wastewater and sewage sludge for fertilization and irrigation. Water Sci Tech. 64(4):871-879.

42. TORRES, L.P; MADERA, P.A.C.; SILVA, L.J.A. 2009. Mejoramiento de la Calidad Microbiológica de Biosólidos Generados en Plantas de Tratamiento de Aguas Residuales Domesticas. Revista EIA. 11: 21-37.

43. USDA. 1954. Diagnosis and Improvement of Saline and Alkali Soils. Chapter 8: Methods of analysis of irrigation water. Agriculture Handbook. 60p. 
44. USDA-NRCS. 2004. Soil Survey Laboratory, Methods Manual, Soil Survey Investigations Report No. 42. USDA y NRCS Natural Resources Conservation Service. United States Department of Agriculture. Washington D.C. 1001p.

45. YANG, W.; LI, Z.; SHU, L.; WANG, J. 2011. Effect of sugarcane//soybean intercropping and reduced nitrogen rates on sugarcane yield, plant and soil nitrogen. Acta Ecológica Sinica. 31(20): 6108-6115.
46. ZALESKI, K.; JOSEPHSON, K.; GERBA, C.; PEPPER I. 2005. Survival, growth, and regrowth of enteric indicator and patho-genic bacteria in biosolids, compost, soil, and land applied biosolids. Journal of Residuals Sci \& Tech 2(1): 49-63.

Recibido: Abril 21 de 2014

Aceptado: Noviembre 24 de 2014

Cómo citar:

Torres-Lozada, P.; Silva-Leal, J.A.; Parra-Orobio, B.A.; Cerón-Castro, V.; Madera-Parra, C.A. 2015. Influencia de la aplicación de biosólidos sobre el suelo, la morfología y productividad del cultivo de caña de azúcar. Rev. U.D.C.A Act. \& Div. Cient. 18(1): 69-79. 